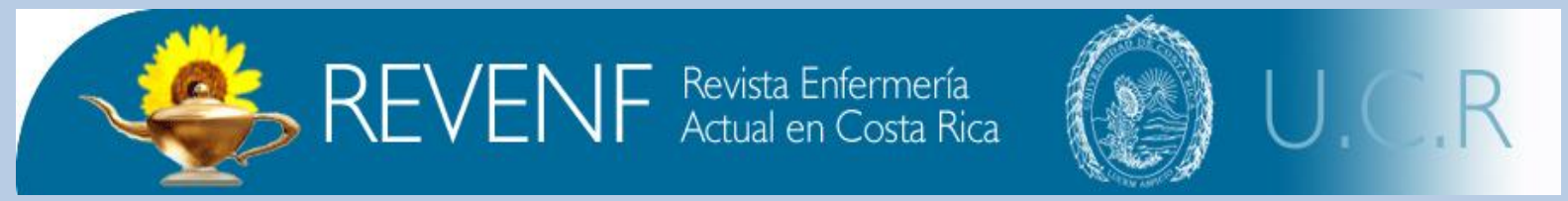

\title{
LA EDUCACION PRENATAL: UNA MIRADA DESDE LA EDUCACION PARA LA SALUD ${ }^{1}$
}

\section{Ensayo}

\section{COMO CITAR}

Rojas Valenciano, Ligia. La educación prenatal: una mirada desde la educación para la salud. Rev. Enfermería Actual en Costa Rica [en línea].2010, No.19 [citado (fecha)]. Disponible World Wide Web: <http://www.revenf.ucr.ac.cr/prenatal.pdf> ISSN $1409-4568$

\section{Ligia Rojas Valenciano ${ }^{2}$}

\section{Universidad de Costa Rica}

Escuela de Enfermería

\section{RESUMEN}

El presente ensayo tiene por objetivo hacer una reflexión en torno a la educación prenatal como parte de la Educación para la Salud. Para ello se toman diferentes enfoques y modelos educativos propuestos a través de la historia cuyo papel ha sido protagónico respecto de la toma de decisiones en distintos contextos sociales. Para este análisis se parte del concepto de educación en general y de Educación para la Salud como fundamento de los enfoques y modelos desarrollados; por otra parte, se presenta también algunos aspectos importantes relacionados con la complejidad en salud para abordar la situación de la Educación Prenatal, a partir de cuatro modelos muy actuales. De esta reflexión se concluye que el abordaje de la Educación para la Salud no responde a un solo enfoque, sino a una mezcla de ellos. Por otro lado, se tiene que la complejidad en salud puede verse como una construcción de un espacio en donde los grupos humanos se relacionan con el ambiente. Finalmente, la Educación Prenatal se ha visto influenciada por dicho enfoque y distintos modelos que promueven un proceso que inicia desde antes de la concepción, continúa a través de ésta y va más allá del parto y el puerperio.

PALABRAS CLAVE: educación, educación prenatal, prenatal, salud.

${ }^{1}$ Fecha de recepción: 14 mayo, 2010

Fecha de aceptación: 30 julio, 2010

${ }^{2}$ Enfermera Obstetra, Doctora en Educación, Magistra en Evaluación Educativa y Profesora Asociada de la Universidad de Costa Rica. Correo electrónico: ligiarojas7@gmail.com 


\title{
PRENATAL EDUCATION: A LOOK FROM THE HEALTH EDUCATION
}

\begin{abstract}
Essay
ABSTRACT

The objective of this essay was to make a reflection on prenatal education as part of Health Education. For this, were extracted the different educational approaches and models proposed through history and have had a role in decision making in social contexts. This analysis of the concept of education in general and Health Education was based in focuses and models developed. It also presents some important aspects of complexity in health. Respect to the situation of antenatal education, were exposed four models very current. It was concluded from this discussion that the approach to health education does not respond to a single approach, but a mixture of them. Besides, the complexity in health is proposed as a construction of a space where human groups are related to the environment. Finally, prenatal education has also been influenced by different models which promotes a process that begins before conception, continues through this, and go beyond birth and the postpartum period.
\end{abstract}

KEY WORDS: education, health, prenatal, prenatal education.

\section{INTRODUCCION}

La educación prenatal, como un proyecto de vida de un grupo humano, así como la planificación puesta en práctica y evaluación, pertenece a la comunidad en su conjunto, razón por la cual el profesional en Enfermería Obstétrica debe estar en constante interacción con todos los actores sociales, relación que exige una participación activa en el cuidado de su salud. Por lo anterior, la Educación para la Salud tiene un papel protagónico que -a través de muchas décadas- se ha nutrido de diversos modelos y enfoques de Educación para la Salud cuyos distintos modos de abordaje se adaptan a las necesidades y posibilidades de los contextos. El presente ensayo pretende evidenciar los diferentes enfoques y modelos de Educación para la Salud y la influencia de que tienen en la Educación Prenatal. El desarrollo consta de cuatro apartados: en el primer apartado se presenta una visión general sobre el concepto de Educación y Educación para la Salud; el segundo apartado reseña algunos modelos de Educación para la Salud, un tercer apartado expone la salud desde el pensamiento complejo y, finalmente, una cuarta parte presenta la situación de la Educación Prenatal. 


\section{Parte. El concepto de Educación y Educación para la Salud.}

De acuerdo con la etimología, la Educación procede morfológicamente y fonéticamente de la palabra "Educare" entendido como conducir, guiar, orientar; por otra parte, semánticamente, se habla del término "Educere" que significa sacar a la luz, descubrir, extraer. Tales conceptos han condicionado la coexistencia de dos modelos conceptuales tradicionales en educación.

Las características del modelo "Educare" oscilan entre lo directivo y lo interviniente. Según este modelo, la Educación es un proceso de socialización que pretende que el individuo adquiera la habilidad de actuar congruentemente con el medio sociocultural al que pertenece. Los programas basados en este modelo hacen énfasis en el producto, es decir, interesalo que lleva al desarrollo de una educación pasiva basada en la memorización, la transmisión de contenidos, con el objetivo de conseguir una cohesión social imponiendo límites a los comportamientos desviados. (Aula virtual

Respecto del aprendiz, se concibe como un receptáculo vacío que, por tanto, el educador debe llenar demostrando autoridad, saber, entre otros aspectos; su función es de transmitir, informar, explicar, repetir; o sea, es de tipo vertical. Por su parte, el estudiante escucha, recibe, asimila, repite.

Un segundo modelo tiende más hacia la posición "Educere": es de tipo asesor en el proceso de adquisión de conocimientos y su objetivo es el desarrollo personal. A diferencia del modelo anterior, éste es un modelo activo en el que el aprendiz es crítico de la realidad sociocultural que le rodea. Concuerda con la posición de aprender a aprender, tomando una actitud activa en el descubrimiento de la realidad. $\mathrm{Su}$ situación sociocultural adquiere especial importancia, por ende, toma en cuenta sus valores y creencias.

Teniendo presente las formas que han enmarcado el concepto de educación antes descritas, se desprenden múltiples iniciativas propuestas sobre la Educación para la Salud. Por supuesto estas ofertas educativas son congruentes con las tendencias y la ideología que ha marcado el desarrollo de los modelos educativos aplicados por los profesionales en su práctica para dar respuesta a los problemas de salud. Es importante resaltar que no existen prácticas educativas puras, para la salud, es decir, que se basan en un solo modelo, por el contrario, se busca una práctica educativa que tenga diferentes matices en sus planteamientos los cuales deben responder a los intereses y gustos al utilizar los métodos y técnicas en la enseñanza.

En la genealogía histórica de la Educación para la Salud, se distinguen dos periodos que marcan el desarrollo de este concepto. (Salleras, 1988). El primer periodo, llamado Clásico, se sitúa antes de la década de los años 70 y se caracteriza por la inclusión de acciones dirigidas a la persona, responsabilizándola del cuidado de su propia salud al modificar conductas insanas individuales. Algunas definiciones del concepto de Educación 
para la Salud, que se retoman en este periodo son las siguientes:

* La suma de experiencias que influyen favorablemente sobre los conocimientos, actitudes y hábitos relacionados con la salud del individuo y comunidad. (Wood, $\underline{1926)}$

* La instrucción de las gentes en materia de higiene, de forma que apliquen los conocimientos adquiridos al perfeccionamiento de su salud (Gilbert, $\underline{1959)}$

* La educación sanitaria es una intervención social que tiende a modificar conscientemente y de forma duradera los comportamientos relacionados con la salud. Ello presupone el conocimiento del patrimonio cultural del grupo y la focalización de sus intereses subjetivos, y requiere la remoción de las resistencias que el grupo opone a la salud (Seppelli, 1970)...Una condición de equilibrio funcional, tanto mental como físico, conducente a una integración dinámica del individuo en su ambiente natural y social. (Seppilli, 1971)

* Proceso que se interesa por todas aquellas experiencias de un individuo, grupo o comunidad que influencian las creencias, actitudes y comportamientos en relación a la salud, así como por los esfuerzos y procesos que producen un cambio cuando éste es necesario para una mejor salud.(OMS, 1969)

En las definiciones antes señaladas se aprecia un elemento común: la modificación de comportamientos en un sentido positivo destacando, según su evolución, las necesidades de dirigir las acciones no sólo a la persona, sino también a la comunidad, y de abarcar en su campo de acción cualquier momento de la historia natural de la enfermedad y no solo el periodo prepatogénico.

Con base en los elementos conceptuales antes mencionados, y tomando en cuenta las condiciones políticas, económicas y sociales de las dos grandes épocas hasta ahora descritas, se ha elaborado un grupo de enfoques y modelos en Educación para la Salud descrito en el siguiente apartado.

\section{Parte. Enfoques y modelos desarrollados en la educación para la salud.}

En el período clásico, los modelos planteados fueron bastante criticados por no considerar los factores ambientales, físicos y sociales. Se señala el enfoque biologicista, de corte tradicional, utilizado en la práctica médica, el cual subraya la información de tipo anatómico-fisiológico, y el enfoque psicologicista, que plantea la importancia del comportamiento individual como responsable de las prácticas de salud existentes, pues existe la posibilidad de adquirir enfermedades.

En el enfoque biologicista se plantearon tres modelos: el biomédico, el informativo y el conviccional (Health Believe Model). El primero de ellos se caracteriza por la utilización de métodos unilaterales en función de la persona enferma. El objetivo es la modificación de comportamientos individuales arraigados. Además, plantea el aporte de conocimientos como un elemento suficiente para conseguir el objetivo propuesto, es decir, la adhesión del paciente al tratamiento. El segundo modelo es más reciente que el primero y 
recoge algunos aportes psicologicistas usados para modificar los comportamientos. Se asemeja al biomédico en cuanto al modo de entender los determinantes del comportamiento, en otras palabras, la falta de información como causa etiológica de la enfermedad. Utiliza el método magistral de información, adoptando el contenido y forma al público receptor, pero considerándolo parte esencial del proceso. Se imparten conocimientos, a todo aquel que desconozca la información al respecto, acerca de comportamientos saludables. Es unidireccional y no estimula la capacidad de razonamiento para aclarar valores y creencias. El tercer modelo es una de las aportaciones más importantes de la Psicología al campo de la Educación para la Salud. Fue formulado por Rosentock y Leventhal en las décadas de los años 50 y 60 . El modelo hace referencia a la expectativa de valores que intenta predecir y explicar el comportamiento en salud. Las creencias influyen en la toma de decisiones en relación a comportamientos promotores o restauradores de salud. En la oferta educativa del modelo en mención cabe destacar la preparación psicológica del individuo para adoptar una decisión de salud; también, las claves o señales para el cambio de comportamientos, denominados estímulo de acción, los cuales pueden ser tanto internos (percepciones del estado somático) como externos (campañas informativas). Por otra parte, asume qué variables sociodemográficas y estructurales pueden influir en las percepciones, pero no las considera causales directas de conductas de salud específicas. (Aula virtual)
El legado de este modelo ha sido la importancia de los estímulos internos (sintomatología), así como las características de la relación terapéutica, útil en la mejoría de los cumplimientos terapéuticos. No obstante, la limitación que tiene es que, al no tomar en cuenta el medio ambiente social en el que se desenvuelve el individuo, no sirve para explicar comportamientos en las personas sanas de la comunidad, en quienes los factores ambientales tienen un papel fundamental. Por otro lado, enfatiza la información para el cambio de actitudes, aunque éstas no garantizan cambios de conducta.

Dentro del enfoque psicologicista, se enmarca el modelo de decisiones en salud, el cual trata de superar las críticas del modelo anterior y recoge aportaciones de la literatura científica respecto de las preferencias del paciente en relación con el cumplimiento terapéutico. Otro modelo detacable en este enfoque es el persuasivo motivacional, también conocido como preventivo, actitudinal o K.A.P. (Knowledge, actitudes, practices) de O`Neil, 1979 (Pirantozzia. L., 2010; p.1). Una de las características más importantes es que presenta la necesidad de incluir la motivación como elemento imprescindible, posterior al proceso informativo necesario para la consecución de determinados hábitos. Metodológicamente, propone una fuente de información que, con suficiente reconocimiento social, transmita la información más adecuada al auditorio con los mejores medios de comunicación.

Sintetizando, en la época clásica, el objetivo fundamental de la Educación para la Salud es la adquisición de conocimientos, actitudes y 
comportamientos saludables, tanto desde lo individual como desde lo colectivo; no se toma en cuenta la modificación del medio ambiente social ni la necesidad de incluir las actuaciones sobre la persona enferma. Actualmente, se sabe que la actuación que se limita al individuo, si no contempla los factores sociales, no logra mantener conductas, aunque se trabaje con una actitud positiva.

Debido a lo anterior, surgen nuevas tendencias en la Educación para la Salud, las cuales recomiendan la inclusión del medio ambiente social inmediato al individuo, por lo tanto, el desarrollo de la educación debe ir dirigida a generar y modificar comportamientos en la persona enferma y a involucrar a la comunidad en todas la fases del proceso educativo. Así es como surgen otras definiciones de educación para la salud para las cuales ésta: Es un instrumento que ayuda al individuo a adquirir un conocimiento científico sobre problemas $y$ comportamientos útiles para alcanzar el objetivo "salud" (Mondolo, 1979)

Es un proceso que informa, motiva $y$ ayuda a saludables, propugna los cambios ambientales necesarios para facilitar esos objetivos, y dirige la formación profesional y la investigación hacia esos mismos objetivos. (National Conference on Preventive Medicine. Sommers A., 1975, citado por Ramón Calvo y otros, s.f.)

En las definiciones antes expuestas sobresalen algunos aspectos como son: el informar a la población sobre la salud, la enfermedad, la invalidez y las formas mediante las cuales las personas pueden mejorar su propia salud. También se señala el motivar a la población como un aliciente para lograr hábitos más saludables; ayudar a adquirir los conocimientos, actitudes y habilidades necesarias para mantener un estilo de vida saludable; incentivar cambios en el medio ambiente que promuevan condiciones de vida más saludables y una conducta hacia la salud más positiva; finalmente, se observa la insistencia en la promoción de la enseñanza, formación y capacitación de todos los agentes de educación para mejorar la salud de la comunidad, incrementar los conocimientos acerca de la manera más efectiva, mediante la investigación y la evaluación, para alcanzar los objetivos propuestos.

Un segundo período corresponde a los enfoques propuestos después de la década de los años 70. Aquí se ubica el enfoque sociologicista, que se caracteriza por prácticas de salud y estilos de vida de la población vistas como un producto social. En su acción, este enfoque minimiza el papel del comportamiento individual y dirige su acción a introducir cambios en el macrosistema social en el que vive la población. Explica de forma clara la Promoción de la Salud y los principios declarados en la Conferencia de Ottawa en 1986. Se orienta a la educación de la comunidad, al fomento, desarrollo y organización de servicios de asistencia y a la organización de la población en defensa de su propia salud.

Desde este enfoque se han desarrollado tres modelos críticos que son:

El modelo político, económico, ecológico llamado también modelo ambientalista, radical y ecológico. En su redacción sostiene que los programas educativos anteriores culpan el comportamiento individual, cuando el verdadero 
responsable es el ambiente social en el que se desenvuelve la persona. Por otro lado, considera la importancia de la clase social como determinante de desigualdad en salud, por tanto, aboga por las clases menos favorecidas puesto que la pobreza es el principal condicionante de enfermedad. El papel del educador para la salud es el de despertar la conciencia de la población respecto de las relaciones existentes entre las condiciones, los estilos y la calidad de vida de la población.

El modelo de desarrollo personal y de habilidades sociales, considera el rol que juegan los factores conductuales no sólo en la prevención, sino también en la intervención terapéutica y rehabilitadora; por otro lado, estudia Lo que se hace o no se hace en una situación dada y cómo el comportamiento determina, de forma directa o indirecta, la salud. El objetivo de este modelo es facilitar a la persona una elección debidamente informada, por ende, sus actividades deben estar orientadas a aumentar la aptitud y competencia conductual individual en la toma de decisiones que afectan al bienestar individual, familiar y social. El aporte sobresaliente de este modelo es su contribución al desarrollo personal y social, también promueve un aumento en la autoestima, dota de habilidades personales y sociales necesarias para desarrollar la autonomía y responsabilidad en la consecución de conductas saludables.

El modelo pragmático o multifactorial es asumido por la Organización Mundial de la Salud (OMS) tras constatar cuán la infructífero es dirigir esfuerzos para cambiar comportamientos en la población si no se trabajan, simultáneamente, las dimensiones económicas y sociales implicadas en el problema de salud. Este modelo combina el modelo persuasivo K.A.P. en la población, aportes del modelo político, económico, ecológico para intervenir sobre los determinantes sociales de salud. Está dirigido a la población sana, con capacidad de autocuidado, y a la población enferma, orientado a la recuperación de la salud.

Hasta el momento, los enfoques y modelos brevemente descritos, empleados en el desarrollo de la Educación para la Salud, salen de la simplicidad y se adentran en la diversidad de nuevas perspectivas y prácticas de salud que van mucho más allá del saber y hacer enfermero. Entre estas nuevas perspectivas está la complejidad en salud y su influencia en la Educación Prenatal, aspecto que se desarrolla en el siguiente apartado.

\section{Parte. La complejidad en salud.}

En las últimas décadas, las perspectivas que abarcan el tema de la complejidad han comenzado a calar fuerte en la cultura y proponen diversas alternativas para salir del universo mecánico de la simplicidad y adentrarse en un multimundo vital en el que son compatibles la estabilidad y el cambio, la unidad y la heterogeneidad. La complejidad implica forjar un marco conceptual completamente distinto que permita concebir sistemas multidimensionales nacidos de una dinámica relacional que incitan el pensamiento y contribuyen con la construcción de mundos de sentido muy diferentes a las que surgen de los modelos de pensamiento basados en oposiciones binarias. 
En relación con el pensamiento, cuidado y atención a la salud, se considera que los enfoques y modelos antes presentados se estructuran a partir de oposiciones dicotómicas fundamentales: cuerpo/mente, individuo/sociedad, humano/naturaleza, normal/patológico, público/privado. Estas polaridades están dentro de la distinción radical entre sujeto/objeto. Por tal motivo, todo proyecto de construcción de abordajes que hagan lugar a la complejidad deben incluir necesariamente el cuestionamiento de la epistemología de base.

Lo anterior no se trata de indicar nuevos lugares en este siglo, sino de construir un nuevo espacio de pensamiento que reúna múltiples formas de producir sentido y construir prácticas profesionales enriquecedoras. La salud, desde una perspectiva de la complejidad, no puede pensarse si no es en referencia al rumbo que fija la misma vida, a los valores que la persona construye y a las prácticas culturales que le dan sentido.

El ser humano es un sistema autónomo auto organizado, producto de una multiplicidad de intercambios que han generado una "unidad heterogénea" emergente con una legalidad propia. Estos sistemas autónomos no son independientes, su autonomía existe en y por las relaciones de intercambio, es una "autonomía ligada", la vida fluye en una red de relaciones y dinámicas transformaciones globales co-evolutivas con el ambiente.

Las concepciones dinámicas dan la oportunidad de concebir la salud como una relación de la persona con su entorno; no es el cuerpo el que se enferma sino el ser humano. La persona humana dotada de emoción y conciencia, afectiva y capaz de conocer, imaginativa y social, inconsciente y entramada, es la que enferma o sana, es la que vive o muere, es un sujeto que se hace en y por los cambios sociales en los que participa y en cuyo ambiente está embebida.

La complejidad en salud es un desafío teórico y pragmático, social e individual, político y ético. Desde el punto de vista epistemológico, requiere una precaución particular; en la actualidad se está atravesando un periodo de transición en el cual si bien es cierto que muchos declaman estar abiertos a un punto de vista que supone a la "salud como bienestar físico, psicológico y social”, son muy pocos lo que han desarrollado enfoques específicos que hagan de esta mirada de la salud algo más que un mero slogan.

Desde esta perspectiva, la salud nunca es un asunto individual, sino que está siempre en la intersección entre el hombre/mujer, su sociedad y su medio ambiente. El enfoque complejo de la salud será posible en tanto trabajen equipos interdisciplinarios en interacción permanente con las comunidades a las que ayudarán a poner en marcha sus planes de salud.

De acuerdo con los conceptos expuestos anteriormente, la Educación Prenatal se debe entender como un proyecto de vida de un grupo humano, la responsabilidad en la planificación, la puesta en práctica y la evaluación: estas últimas pertenecen a la comunidad en conjunto. Esta perspectiva consiste en una red de relaciones dinámicas entre el grupo humano y el ambiente; él o la profesional en Enfermería Obstétrica está en 
constante interacción con todos los actores sociales, lo cual exige una participación activa en el cuidado de su salud. No obstante, se debe tener presente que los modelos multidimensionales (complejos) no pueden sostenerse en el marco de las instituciones actuales de salud las cuales se caracterizan por una rígida organización jerárquica y una división del trabajo, el saber y la responsabilidad fundada en modelos y sistemas de salud burocratizados $y$ plagados de intencionalidades lucrativas, rentabilidad y eficacia administrativoeconómica. No obstante, en muchos lugares se van desarrollando modalidades de trabajo cada vez más distintas y complejos modos de abordaje en salud que se adaptan a las necesidades $y$ posibilidades contextuales.

El pensamiento complejo, impulsado por Edgar Morin, comienza a cobrar una estructura articulada de conceptos. Surge donde el pensamiento simplificador falla, es un pensamiento multidimensional en donde no se puede aislar al objeto de estudio de su contexto, de sus antecedentes, de su devenir. (Morin, $\underline{1976 / 1988)}$

La complejidad es el tejido de eventos, acciones, interacciones, retracciones, determinaciones y azares que constituyen nuestro mundo fenoménico. Este pensamiento complejo no resuelve en sí los problemas, pero constituye una ayuda para la estrategia que puede resolverlos si dejamos en la base la simplicidad en la atención y educación en salud y si los/las profesionales en salud proponen formas distintas de afrontar los problemas socioeducativos y de salud desde una visión más dinámica e interrelacionada, tomando en cuenta la individualidad y a la vez la cultura de los grupos.

Los enfoques y modelos tradicionales de abordaje de la Educación para la Salud no han sido muy exitosos, puesto que cada año se observa que, de un estimado de 120 millones de embarazos que ocurren en el mundo, más de medio millón de mujeres mueren como consecuencia de complicaciones durante el embarazo o el parto; el 99\% de ellas vive en países en desarrollo; el 50\% de millones de mujeres sufren enfermedades o discapacidades serias relacionadas con el embarazo y, por lo menos, 1.2 millones de recién nacidos mueren por complicaciones durante el parto. Estas cifras, llevan a repensar en los derechos humanos relacionados con la maternidad segura: el derecho a la vida, a la libertad y a la seguridad, a la información y a la Educación en Salud, a los valores humanos y cambios de comportamiento individual y social.

En Latinoamérica y el Caribe, 15.000 mujeres murieron por embarazo y parto para el año 2005. En ese sentido, Costa Rica ha sufrido un retroceso, ya que en el año 2008 fallecieron 25 mujeres antes, durante y después del parto, siendo los trastornos hipertensivos las principales causas de mortalidad materna.

En la región, varían las condiciones de salud y la capacidad de respuesta a las necesidades. No obstante, el elemento común en toda la región es el que la mujer lleva la peor parte en términos de inequidad.

Toda mujer tiene el derecho fundamental a un embarazo y a un parto seguro, en fin, a una maternidad segura. Pero es palpable que el embarazo, el parto y el 
puerperio siguen siendo procesos altamente riesgosos. La discriminación por razones de género, culturales, étnicas, económicas y territoriales, constituye una barrera al acceso a servicios de salud, tales como la Educación Prenatal y a la atención calificada del embarazo y el parto.

Para el año 2000, hubo en Latinoamérica una mortalidad materna de 129 por cada 100.000 nacidos vivos. Se brega no solo con la injusticia de estos acontecimientos sino también con graves repercusiones emocionales, sociales y económicas para la familia y toda la comunidad.

La pérdida de la madre reduce las posibilidades de supervivencia de su recién nacido, el desempeño escolar de los hijos e hijas sobrevivientes, pérdida de productividad económica y de los ingresos familiares y comunitarios. Las personas se enfrentan ante la deficiente calidad de los servicios que tiene que ver tanto con los contenidos de la atención como con los procesos reales involucrados en su aplicación; diariamente se realizan prácticas en salud y se siguen patrones sin sustento en una práctica clínica basada en la evidencia científica.

La modificación de las prácticas en salud, o sea, pasar de la simplicidad que ofrecen los enfoques y modelos tradicionales de educación para la salud, a un pensamiento complejo, requerirá de todos los actores sociales de capacitación, modificación de patrones de comportamiento, buenas actitudes, recursos bien canalizados, y una adecuada disposición de hacer las cosas de la mejor manera.
A continuación, se expone una revisión sobre las prácticas que se han promovido para llevar a cabo la educación prenatal; sin embargo destaco que falta mucha investigación que evidencie realmente cual estrategia es la ideal o mejor para disminuir las estadísticas señaladas en este apartado.

\section{Parte. La situación de la Educación Prenatal.}

Por décadas, el abordaje de la Educación Prenatal ha sido confundido con el desarrollo de un curso de preparación para el parto, caracterizándose éste por unas escasas lecciones elaboradas y ejecutadas desde el enfoque biologicista y psicologicista de la Educación para la Salud, por tal razón los resultados son poco exitosos.

Una revisión sistemática sobre el tema incluyó la siguiente: búsqueda:

- en el registro de ensayos del grupo COCHRANE de embarazo y parto en abril del 2006,

- CINAHL de 1982 hasta abril 2006,

- ERIC 1984 hasta abril del 2006,

- EMBASE desde 1980 hasta abril del 2006

- PSYCINFO desde 1988 hasta abril del 2006,

- JOURNAL OF

PSYCHOSOMATIC RESEARCH desde 1956 hasta abril del 2006.

Se incluyó en esta revisión, 9 ensayos controlados aleatorizados con participación de 2284 mujeres, relacionados con cualquier tipo de programa estructurado, ofrecido a los padres durante el embarazo, brindado por un educador con formación relacionado 
con embarazo, parto o la paternidad. Las intervenciones educativas podían haberse suministrado en grupos o de manera individual; se evaluó la calidad de los ensayos y extrajeron datos a partir de informes publicados.

La conclusión de los investigadores revisores fue que se desconocen los efectos de la Educación Prenatal general ya sea para la etapa del parto, la paternidad, o ambos. La Educación Prenatal individualizada, con el objetivo de evitar un parto por cesárea repetido, no aumenta la tasa de partos vaginales después de una intervención por cesárea. Por lo tanto, los efectos de la Educación Prenatal siguen siendo en gran parte desconocidos. Se requiere realizar una investigación adicional para asegurar maneras efectivas que ayuden a los profesionales de la salud a apoyar a las mujeres embarazadas y a sus parejas en la preparación para el nacimiento y la crianza, con el fin de que los recursos usados suplan las necesidades de los padres y sus recién nacidos. (Gagnon AJ, $\underline{\text { Sandall J. 2007) }}$

Por otro lado, otros estudios también apuntan la falta de investigación (Lauzon

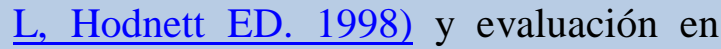
relación con la Educación Prenatal y la preocupación real de principios de crianza de los hijos e hijas en la infancia, etapa que influye en el desarrollo.

Se han desarrollado una diversidad de programas de Educación Prenatal con diferentes enfoques, algunos de ellos son los siguientes:

\section{$\Rightarrow$ Educación prenatal natural.}

$\Rightarrow$ Sistema electrónico para la educación prenatal Baby Plus.

$\Rightarrow$ Programa educativo prenatal con énfasis en la promoción del apego in útero de Chile.

\section{$\Rightarrow$ Aprendizaje holístico superaprendizajel acelerado.}

En cuanto a la Educación Prenatal Natural, brindan la siguiente definición:

La educación prenatal es una prevención real y fundamental y extensa, se trata de una toma de conciencia colectiva en todos los países. Es transformadora de los futuros padres, y de los jóvenes, que adquieren una nueva conciencia de sí mismos, de la vida, y de su papel de educadores. Es formadora de un ser abierto gracias al amor recibido, y fortificado por el respeto, el reconocimiento y la confianza que le habrán impregnado celularmente. Es positivizada por los padres, participa en la génesis de la salud física y psíquica del ser que se forma. Esta educación es la prevención más fundamental de la violencia y de otras disfunciones. (Asociación Nacional de Educación Prenatal)

Por otro lado, el sistema Baby Plus, consiste en 16 lecciones que se imparten entre las 18 y 32 semanas de embarazo. Estimula el aprendizaje y potencial creativo de los hijos e hijas durante la gestación y nacimiento; tiene una concepción integral del ser humano desarrollado emocional, mental y socialmente.

El programa educativo que se imparte en Chile tiene por objetivo promover la adopción de conocimientos respecto de las etapas psico-emocionales del embarazo, generar instancias de trabajo individual y grupal que faciliten la elaboración psicológica de la experiencia del embarazo y facilitar el descubrimiento 
de las influencias que el sistema emocional familiar tiene sobre la experiencia del embarazo y la futura relación madre-hijo.

Finalmente, el superaprendizaje, o aprendizaje holístico, se refiere a un conjunto de técnicas y estrategias dirigidas a desarrollar el proceso de aprendizaje en forma holística. En este sentido, permite conocernos más a nosotros mismos, asumir el aprendizaje con compromiso, disciplina y con amplio sentido de lo que es aprender. (Lozanov, G., 1985)

El sistema fue desarrollado por el científico Georgi Lozanov, psiquiatra y parasicólogo búlgaro en la década de los años $60 . \quad$ Creó la sugestopedia, investigación basada en el uso de los recursos de la mente humana, como forma novedosa y poderosa de aprender y expandir la memoria a través del estudio de las técnicas del Yoga Raja y Yoga Mental, las cuales son desarrolladas por los Yoghis; con ellas se logra mejorar la capacidad de alcanzar la súper memoria o hipermnesia usando la respiración consciente y la relajación, de manera que descubrieron un estado óptimo de aprendizaje.

En Ucrania, mientras hacía investigaciones para su doctorado, descubrió que en los hospitales búlgaros y rusos se escuchaba música barroca del siglo XVII, hecha por compositores como Vivaldi, Telemann y Bach. Luego, agregó a su investigación la música clásica de Mozart, Beethoven y Brahms, cuyos efectos en la mente y la memoria eran relajantes, armónicos y poderosos. Descubrió también que esta música ayudaba a la regulación del ritmo cardíaco y la presión sanguínea.

Este sistema de aprendizaje óptimo consiste en ubicarse en el mejor estado mental corporal relajado, a través de respiraciones con el uso de la música para expandir la memoria y suministrarle energía al cerebro, de manera que se absorba la información de una forma pausada y rítmica, con el estrés necesario para estar motivado, relacionando la respiración controlada y consciente, la cual se considera como un acto vital realizado en la mayoría de las personas de forma inconsciente, por tanto, no son capaces de captar la importancia de este proceso. Entre los beneficios de la respiración están: liberación de cargas inútiles, purificación del organismo, revitalización de fuerzas, expulsión de todo aquello que oprime, intranquiliza, deprime y enferma, además ayuda a concentrarse y a fijar la atención.

Respecto de la respiración asociada al proceso de enseñanza y aprendizaje, se afirma que:

Para lograr el superaprendizaje la respiración es esencial, pues se aprende a respirar rítmicamente, lo cual nos dota de mejor oxigenación, mejor vida; cuanto mejor oxigenado tengamos nuestro cerebro, nos conservaremos más claros, más lúcidos, más eficientes y por tanto más inteligentes. Aprender a respirar es básico, y al coordinar la entrada y la salidad del aire, con contenidos a aprender, tendremos resuelta una de las claves del superaprendizaje." (Sambrana, J. 2001; s.p.)

Otro elemento importante es la relajación, definida como un estado del cuerpo y de la mente en el que nos encontramos sin estrés, por lo tanto, nos lleva a un estado de conciencia y la energía del cerebro es empleada en la 
función que está desempeñando en un momento determinado, sin desperdiciarla, proceso que dota a la persona de un gran potencial para aprender lo que desea. Cuando se está relajado se puede eliminar tensiones innecesarias, mejorar el funcionamiento fisiológico y aumentar la sensación de tranquilidad y paz.

Por otro lado, la música constituye una herramienta fundamental para la aplicación de la técnica del superaprendizaje. Pero no todo tipo de música contribuye a ello: la música ideal es la producida en la época o período barroco, la hindú y la música contemporánea conocida como nueva era aplicable a este efecto (ayuda a la producción de las ondas alfa). La música entona al cerebro y al mismo tiempo le dispone para introducirse en el proceso de enseñanza. $\mathrm{Al}$ respecto, en educación prenatal se habla sobre el canto prenatal. En esta técnica se señala que el niño o niña en gestación recibe las vibraciones sonoras a través de la conducción nerviosa (la piel) y, después del quinto mes de gestación, se agrega la conducción ósea. El marco teórico-metodológico del canto prenatal es la Psicofonía, técnica de trabajo vocal y corporal creada por Marie-Louise Aucher. (Vizcaino, P.:s.f.)

La visualización es una técnica que ayuda a disminuir el estrés y alcanzar un estado óptimo para aprender. Con visualizaciones y algunos ejercicios, se ayudará a la madre a tomar conciencia de cómo hemos vivido nuestro propio nacimiento y a transformar aquellas decisiones de modo que el niño o la niña tengan la oportunidad de nacer en armonía, libres de cargas innecesarias.
El superaprendizaje considera que todos los elementos antes mencionados deben relacionarse y aplicarse dentro de un ambiente que reúna condiciones específicas, dada su importancia en el proceso de aprendizaje; el aula se debe entender como un espacio multidimensional, que presenta formas y características asociadas a los intereses y necesidades de los aprendices. El ambiente en el aula no solo está conformado por las relaciones y tipo de comunicación que se establezcan entre los sujetos del proceso educativo, también es determinante la forma en que está organizada el aula de clase, es decir, los recursos con que se cuenta. Se recomienda, por tanto, un clima de apertura y diálogo a través de una comunicación asertiva $\mathrm{y}$, a su vez, organizar el espacio de aprendizaje en pequeños grupos, en forma de $\mathrm{U}$ o en círculos, para que la comunicación sea cara a cara de modo que se pueda mirar la expresión del rostro.

Otras técnicas que utiliza el superaprendizaje es el color: el color que tengamos en el entorno puede influir en el comportamiento ya sea produciendo relajación o irritación, serenidad o estimulación. El color se ha asociado con la salud: el Dr. Szent-Gyorgyi, ganador del premio Nóbel por el descubrimiento de la vitamina $\mathrm{C}$, descubrió que muchas enzimas y hormonas tienen color y son sensibles a la luz, de hecho, cuando son estimuladas con diferentes colores, pueden sufrir cambios moleculares que alteran sus colores originales. Por lo tanto, se recomienda estudiar los colores utilizados en la decoración de los espacios destinados al aprendizaje, para estimular aspectos como la creatividad o 
para generar un ambiente sereno y tranquilo.

Finalmente, la aromaterapia es una técnica muy utilizada en el aprendizaje holístico. En la actualidad, los aromas son objeto de interés en los círculos médicos más acreditados. El Dr. Gary Schwartz, profesor de Psiquiatría y Psicología de la Universidad de Yale, utiliza el poder curativo del aroma para el tratamiento de muchas enfermedades y alteraciones. De todos los aromas que ha analizado, parece ser que el mejor para reducir el estrés es un olor a manzana que recuerda a la sidra o al pastel de manzana. Lopategui (s.f.) indica que en un estudio realizado por Schwartz, la fragancia aromática a manzana hizo que la presión arterial sistólica de las personas analizadas descendiera de 3 a $4 \mathrm{mmHg}$.

Todas las formas antes descritas, aplicadas a la Educación para la Salud y propiamente a la Educación Prenatal, estimulan el aprendizaje y potencial creativo de los hijos e hijas durante la gestación y el nacimiento y se prolonga durante todo el desarrollo del ser humano. El niño o niña antes de nacer ya tiene 40 semanas de vida, por lo cual, todo lo que haya sentido, vivido, experimentado en el útero $\mathrm{y}$, principalmente, a través de su madre y de su entorno, se graba en su subconsciente al punto de que esos esquemas de comportamiento le influenciarán en el desarrollo de su personalidad. (Vizcaíno, P.:s.f.)

\section{CONCLUSIONES.}

-Se han descrito dos periodos importantes en la Educación para la Salud que enmarcan enfoques y modelos empleados. Sin embargo, las formas de abordaje de la
Educación para la Salud generalmente no responden a un solo enfoque, sino a una mezcla de ellos.

-La complejidad en salud es una forma de pensamiento y actuar que promueve una red dinámica de interacciones sociales, económicas, políticas, culturales, entre otros aspectos. No pretende ser un nuevo modelo sino más bien la construcción de un espacio en donde se relacionan los grupos humanos con el ambiente o su contexto.

-La educación prenatal es un proceso que inicia desde antes de la concepción, continúa a través de ésta y va más allá del parto y el puerperio. Debe formar parte de los servicios y atención que se les brindan a las personas. Este proceso requiere de mucha investigación que ayude al mejoramiento de la calidad de la atención en salud.

\section{REFERENCIAS}

-Asociación Nacional de Educación Prenatal http://anep.pangea.org/queeslaeducacionprenatal.h tm Consultado 8 de octubre 2009.

-Aula virtual. Enfermería y educación para la salud. Consultado el 10 setiembre 2009. www.fuden.es/FICHEROS.../aula/aula acredit_e ps_oct04.pdf

-Baksh Laurie y otros (s.f.) La Educación prenatal en UTAH

http://health.utah.gov/mihp/pdf/Prenatal\%20Educ ation.PDF consultado 5 de mayo 2010.

\section{-El superaprendizaje}

http://www.monografias.com/trabajos11/super/su per.shtml

-Gagnon AJ, Sandall J. Individual or group antenatal education for childbirth or parenthood, or both. Cochrane Database of Systematic Reviews 2007, Issue 3. Art. No.: CD002869. DOI: 10.1002/14651858.CD002869.pub2. 
-Gilbert, J. (1959). L'educatión sanitaire, theorie et practique. Paris: Masson et Cie

-COMITÉ DE EXPERTOS DE LA OMS EN EDUCACION SANITARIA. (1969) Planificación y Evaluación de los Servicios de Educación Sanitaria. Serie de informes técnicos No. 409. Geneve: O.M.S.

-Lauzon L, Hodnett ED. Antenatal education for self-diagnosis of the onset of active labour at term. Cochrane Database of Systematic Reviews 1998, Issue 4. Art. No.: CD000935. DOI: 10.1002/14651858.CD000935.

-Lopategui Corsino E.(s.f.) Estrés: Concepto, Causas y Control. Universidad Interamericana de PR - Metro, Facultad de Educación, Dept. de Educación Física. San Juan.

-Lozanov, G. (1985). Sugestología y supra aprendizaje. Bogotá: sin editorial.

-Mondolo, M.A. (1979) Educación Sanitaria, comportamiento y participación, II pensiero scientifico, compilación CAPS, 8, pp.39-58.

-Morin, E. (1976-1988). Introducción al pensamiento complejo. Madrid: Editorial ESF

-Pirantozzia, L.La educación para la salud un abordaje en el trabajo de enfermería con las comunidades. Revista electrónica de Portales Médicos.comhttp://www.portalesmedicos.com/pu blicaciones/articles/2331/2/La-educacion-para-lasalud-un-abordaje-en-el-trabajo-de-enfermeriacon-las-comunidades Consultado 14 julio 2010.

-Programa educativo prenatal con énfasis en la promoción del apego in útero de Chile: Chile Crece Contigo.

-Sistema Baby plus.

http://www.babyplus.com.mx/ciencia.html.

Consultado el 15 setiembre 2009.

-Ramón Calvo y otros (s.f.) Educación para la salud. Una estrategia para cambiar los estilos de vida: El Ejemplo del tabaquismo. Consultado el $15 \quad$ setiembre 2009. www.fgcasal.org/aes/docs/Calvo.pdf
-Salleras, L.(1988). Educación sanitaria: principios, métodos y aplicaciones. Madrid: Díaz de Santos. Madrid.

-Sambrano J. (2001) Súper aprendizaje. El placer de aprender a aprender. Caracas: Ed. Alfadil.

-Seppelli, A. (1970). Heath Education and Protection of Heath, en Lotta tuberc, 40 (1) pp 4247.

-Vizcaíno, P.(s.f.) La estimulación intrauterina. Conferencia http://www.waece.com Consultado 5 setiembre del 2009.

-Wood, T.D. (1926) Fourth Yearbook of the Department of Superintendent of the National Educational Association. Washington: National Educational Association. 